\title{
FORUM
}

\section{Collision Avoidance by Manœuvre}

\author{
E. S. Calvert
}

In the Interim Report of the Institute's Collision Regulations Working Party (Journal, 23, 448), it was stated in the preliminary that Dr. Calvert was not able to subscribe entirely to propositions ( 1 ) to (19). Dr. Calvert here states his views.

My disagreement with the interim report is concerned only with the propositions and principles at the end. The body of the report I regard as masterly in the circumstances.

In good visibility mariners have in practice chosen anti-clockwise rotation of the sight-line as the basic rule of the sea, but have not made it mandatory in the Regulations. In bad visibility, when the sightings are by radar, they have so far not chosen either of the two possible rotations. My first objection to the propositions and principles in the report is that the choice is still not made, and that this leads to unnecessary verbiage, and confusion between ends, means and methods. The 'new approach', with which I am credited in the preliminary, starts with the proposal that any new rules should lay down, without equivocation, that, except in emergencies, the tactics of evasion should be such that when the parties return to course and speed, anti-clockwise rotation will occur. This rotation then becomes the overall strategic aim of the whole procedure of evasion. The tactics and information necessary to achieve this aim are complex, and sometimes almost incredible, and these, I suggest, would be best separated off and put in what one might call a 'Seaway Code', on the analogy of the Highway Code. It should also be made clear that the new Code is for traffic systems, i.e. systems in which each party is holding a definite course and speed to which each wishes to return with minimum disturbance. Another point which needs to be made clear at the outset is that rotation of the sight-line is the only criterion for avoidance in a traffic system; this disposes of the idea, often subconscious, that there is a second criterion, namely, increase in separation. Separation without rotation simply produces what may be called a 'yo-yo', i.e. the initial situation repeats itself when the parties return to course and speed, and the separation begins once more to close. This means that it must always be borne in mind, particularly when discussing threats on or near the beam, that an encounter is not over until each party has returned to course and speed.

We can now discuss the tactical aspects of the problem, and it is here that the real difficulties arise. The mathematical work done at R.A.E. around 1960 removed some of these difficulties by showing that the manœuvres which produce a given rotation depend only on the compass bearing of the threat, thereby making it possible for the mariner to refer to simple diagrams instead of struggling with vector geometry. Since the two parties read the same diagram at different places, it follows that all the mancuvres shown on it, if they are to be additive, must be of the same sign, i.e. must produce the same rotation. Nevertheless, several diagrams have been put forward in which the mancuvres recommended for threats on or near the port beam are negative, all the rest being positive. These 
diagrams therefore conflict with proposition (7). This departure from anticlockwise rotation is, of course, an attempt to cope with a double contingency, i.e. that own vessel is unable to increase speed, and that the other vessel does not manœuvre. In my view, the solution is not to meddle with the basic strategic aim, but to treat this case as an emergency, and work out suitable tactics. After all the fact has to be faced that every limitation in manœuvrability implies a limitation to the contributions which can be made in certain circumstances. Another example occurs in the existing Regulations in that the onus to avoid may fall on the slow vessel; this means that, if the speed ratio is high, the onus is, in effect, on a slowly moving obstruction. The existing Regulations therefore begin to fail as speed ratios rise; the only way of avoiding this would be for all the slow vessels to be equipped with predictive systems of very high accuracy, which is, of course, impracticable. With the proposed new system this difficulty does not arise, because there is always an onus to manœuvre on the faster vessel; hampered vessels therefore do not need to be specially catered for.

Once it was realized that both the initial situation and the manouvres have signs and magnitudes which obey the ordinary rules of arithmetic, it was obvious that many conceptual and practical difficulties could be removed by simply dropping the ideas of 'responsibility' and 'privilege', and replacing them with the concept that the evasive mancuvre is the beginning of a continuing process by which a contribution to the miss distance is progressively built up. In other words, the times for which the manœuvres are held by each party separately are taken into account, as they must be in any complete and rational theory. I have the impression that the theory, as taught in Schools of Navigation and textbooks, often fails to do this, i.e. the difficulty is evaded by taking as illustrations only those simple cases in which the tracks of the two vessels converge after the evasive manœuvres. Indeed the fact is that general (and fallacious) arguments, based on simple cases, are the bane of all discussions on collision avoidance.

This new concept proved absolutely invaluable for analytical purposes, and also led to the so-called 'Calvert display'. In this display, observers can, at any moment, see the sign and magnitude of their own and the other party's contribution, and can watch the miss distance grow. When it has grown to a value which one party regards as acceptable, he returns to course and speed. The miss distance then continues to grow, but at a lesser rate, until the other party also returns to course and speed, so both parties, at all times, can see just what each is doing. These ideas were available in the original papers published around 1960; they were further amplified and explained in four papers submitted to the Working Party, but they were not discussed and are not reflected in the propositions and principles.

We now come to a dilemma which springs from the errors inherent in the fact that a risk of collision is assessed by watching the compass bearing of the approaching vessel. The mariner naturally wishes to manœuvre at long range, particularly when using radar, because he cannot be sure of what the other party will do. However, if he does so, he is taking action on information whose badness increases as the square of the range. Since the other party is in the same state of uncertainty, we now have the probability that both parties will act on bad information. The result is that both are likely to be manceuvring in an attempt to increase a miss distance which, had they delayed, would have turned out to be acceptable. This is very undesirable in a traffic system, if only because it may start a chain reaction, a sort of dodgem car situation. As an example of how bad this information can be, let us suppose that the smallest rate of change of bearing 
which a particular observer can 'appreciate' is $3^{\circ}$ per mile of relative track. Now the relationship between miss distance $m$ and range $R$ for this predictive accuracy is $R=4.5 \sqrt{ } \mathrm{m}$, as plotted on Fig. I. At a range of 10 miles, the spread in the miss distance is also I o miles, which means that our observer may, in the worst case, assess as a collision a situation in which the actual initial miss distance is -5 miles. If he manœuvres at just over 10 miles on this assessment,

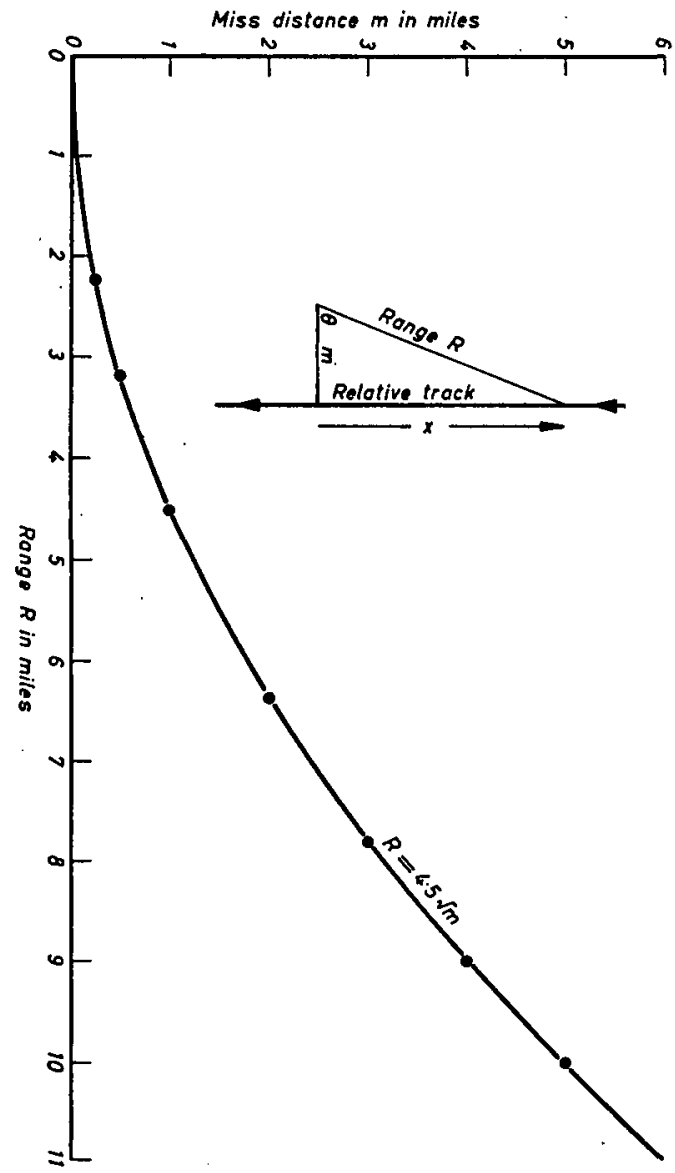

FIG. I. Range and miss distance for a value of $d \phi / d x$ of $3^{\circ}$ per mile

his manœuvres will be positive as required by the basic rule. Another observer, with a more accurate predictive technique, might assess the miss distance as between, say, -3 and -7 miles; he may then manœuvre negatively in an attempt to 'take early and substantial action to avoid a close quarter situation'. It seems to me, as a human factors engineer, that with existing displays the outcome of these mancuvres is likely to be fortuitous, whatever the rules. In other words, the phrase 'radar assisted collision' is not a joke; it is a grim reality. If an actual example is needed, one has only to read the verbatim account of the collision between the Andrea Doria and the Stockholm.

Those who do not believe in mathematicians can obtain pointers towards the $10-N$. 
solution by observing the tactics adopted by a dog or pony which wishes to keep out of his master's way. These animals are highly manœuvrable, and because of this can afford not to manceuvre early, i.e. they can afford to wait until the information about their master's action is very nearly perfect; this, in turn, enables them to dispense with rules. If one watches a flock of starlings circling around preparatory to landing for the night, one never sees two birds collide, which again indicates the extreme importance of good mancuvrability combined with short manœuvring ranges, particularly in traffic. I therefore suggest that what I have called the Seaway Code must try to establish useful working relationships between predictive accuracy, manouvring range, manouvrability and speed ratio. Hitherto this need has been evaded or obscured by basing the Regulations on responsibility, and by question-begging phrases. If the kind of weasel wording used in the present Regulations is carried over into the new, as it is in the propositions and principles at the end of the interim report, then I believe that the annual collision rate will continue at substantially its present level.

The encounter described above is only one example of the general dilemma of what do to when the initial situation is assessed as substantially negative. In an uncontrolled traffic system, about half of the initial situations will be negative, so the problem must be faced. It may well be that no complete solution is possible, but I think that a capacity to exchange signals about the sign of the initial situation, the adoption of a display which separated the contributions and showed their sign and instantaneous magnitude, and a Seaway Code which dealt in realities instead of verbiage, would go far towards reducing the annual collision rate.

In the meantime the best hope of reducing the collision rate, particularly in narrow waters, is to use the sidestep manouvre, i.e. a large alteration made at short range, and held for a short time. With this manœuvre the miss distance on the resumed courses is independent of the manœuvring range, provided only that one of the vessels has time to execute the manœuvre. (It is, of course, better if both vessels leave themselves time to manœuvre.) The condition for a safe sidestep is, of course, the adoption, without exception, of a convention as to the rotation of the sight-line. The sidestep mancuvre is, as we all know, the manœuvre used on land, and every motorist realizes, without having to think about it, that the contribution it makes to the miss distance is independent of range, provided only that he leaves himself enough time to complete the manœuvre. This is why manœuvrability is important, and not, as all mariners seem to assume, earliness in manœuvring. Indeed, if vessels were highly mancuvrable, they could almost touch before they manouvred, provided always that both obeyed the convention. This is why the refusal to adopt the anti-clockwise convention, or even to discuss the papers in which the sidestep manœuvre has been described, seems to me to be the blunder of blunders in the marine world at present. My experience of this Working Party has left me with the impression that these misconceptions have, in the past, been fostered both by officialdom and by the Schools of Navigation and that this is likely to continue for the foreseeable future. Indeed, the ordinary mariner seems to me to have had a very raw deal in that the guidance he has been given as to how to avoid collision has usually been fundamentally fallacious. It is therefore fortunate that the Institute provides a Forum in which views, such as those expressed above, can be put forward.

The argument is illustrated in Fig. 2. 


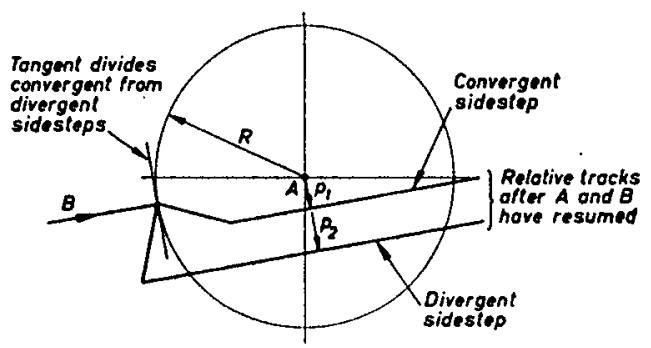

FIG. 2. Convergent and divergent sidestep mancuvres

The perpendiculars, $p_{1}$ and $p_{2}$, to the relative tracks after both vessels have resumed are obviously independent of the mancuvring range, $R$. In the divergent case, $P_{2}$ is always the nearest approach distance after both vessels have resumed course and speed. Divergency commonly arises when the closing speed is moderate or low, i.e. when the threat is abeam or astern. (See diagrams in this Journal, 14, 397, 1961.)

In the convergent case, $p_{1}$ is the miss distance if the evasive mancuvre has been executed in 'proper time'. If not, then there may be a smaller miss distance on the relative track of $B$ before $A$ and $B$ resume; but if this is so, then resumption has been delayed longer than was necessary. This delay is highly undesirable in narrow waters with congested traffic.

\section{A System of Rules for Preventing Collisions at Sea}

\section{Rear-Admiral J. García-Frías}

The following proposed system of rules was presented to the Collision Regulations Working Party of the Institute by Admiral Garcia-Frías and is referred to in the January issue of the Journal $(24,56-9)$.

Ir is evident that the revision of the Collision Regulations would only be effective with a system of Rules able to cope with all situations that may arise at sea. The system must include all that it is necessary and sufficient to solve the collision problem in the easiest and most effective way and consequently it must apply both in clear weather and in restricted visibility using radar; differences between these situations being defined, if necessary, in every Rule.

The two sets of Rules the author has proposed in previous papers ${ }^{1,2}$ were separately intended to solve these problems and an attempt is now made to recast both sets in the following system. It also includes manœuvring rules, which are not however so essential from the point of view of safety as those based on the organic aspect ${ }^{3}$ of the collision problem. 